\title{
Significance of the Acromiohumeral Distance on Stress Radiography for Predicting Healing and Function after Arthroscopic Repair of Massive Rotator Cuff Tears (231)
}

\author{
Young Dae Jeon, $\mathrm{MD}^{1}$, Hyeon Jang Jeong, Joo Han $\mathrm{Oh}, \mathrm{MD}, \mathrm{PhD}^{2}$ \\ Ulsan University College of Medicine, Ulsan University Hospi ${ }^{1}$ Seoul National University ${ }^{2}$
}

Objectives: Decreased acromiohumeral distance (AHD) is commonly detected in massive rotator cuff tears (mRCT). Most studies evaluating fixed humeral elevation have used preoperative or postoperative standardized radiography, and not stress radiography. We aimed to evaluate the role of preoperative AHD using stress radiography for healing and function after arthroscopic repair of mRCT.

Methods: We analyzed the data of 113 patients who underwent arthroscopic repair of mRCT, whose postoperative cuff integrity was evaluated using magnetic resonance imaging at 1 year and whose functions were evaluated at a mean of $34.9 \pm 17.8$ months. Fortyseven patients showed healing failure. Propensity score matching (1-to-1) was performed between the healed and healing failure groups. 38 patients in each group were matched in the final analysis. We defined AHD and AHD_stress as the shortest distances from the inferior acromion to the superior humerus on standard anteroposterior and stress radiography (5.4 kg weight applied inferiorly in a neutral position), respectively. AHD difference (AHD_diff) was defined as the difference between AHD and AHD_stress.

Results: There was no difference in the mean preoperative AHD between the healed $(7.5 \pm 2.0)$ and healing failure groups $(6.9 \pm 2.2$, $p=0.234)$. AHD_diff was significantly higher in the healed $(4.4 \pm 2.1 \mathrm{~mm})$ than in the healing failure group $(3.0 \pm 2.0 \mathrm{~mm}, p=$ 0.002: cutoff, $3.2 \mathrm{~mm})$. Patients with AHD_diff $\geq 3.2 \mathrm{~mm}$ showed lower healing failure $(28.9 \% \mathrm{vs} 71.1 \%, p<0.001)$ and higher functional scores than those with AHD_diff $<3.2 \mathrm{~mm}$. AHD_diff was higher in the American Shoulder and Elbow Surgeons (ASES) $\geq 80(4.9 \pm 1.9 \mathrm{~mm})$ than in the ASES $<80$ group $(3.1 \pm 2.1 \mathrm{~mm}, p=0.024)$. Only postoperative AHD was related to postoperative functions (cutoff, $4.8 \mathrm{~mm}, p=0.009$ ) in the healing failure group.

Conclusions: The AHD_diff measured using preoperative stress radiography can be another predictor of rotator cuff healing and function after arthroscopic repair of $\mathrm{mRCT}$ and would be helpful to determine appropriate treatment strategies.

The Orthopaedic Journal of Sports Medicine, 9(10)(suppl 5)

DOI: $10.1177 / 2325967121$ S00339

(C) The Author(s) 2021

This open-access article is published and distributed under the Creative Commons Attribution - NonCommercial - No Derivatives License (https://creativecommons.org/licenses/by-nc-nd/4.0/), which permits the noncommercial use, distribution, and reproduction of the article in any medium, provided the original author and source are credited. You may not alter, transform, or build upon this article without the permission of the Author(s). For article reuse guidelines, please visit SAGE's website at http://www.sagepub.com/journals-permissions. 